Musées, Patrimoine et Culture scientifiques et techniques

$176 \mid 2018$

mars-avril 2018

\title{
Les Parlements de sciences, outils au service des débats science-société entre jeunes
}

\section{Amandine Duluard}

\section{OpenEdition}

Journals

Édition électronique

URL : http://journals.openedition.org/ocim/1999

DOI : 10.4000/ocim.1999

ISSN : 2108-646X

Éditeur

OCIM

Édition imprimée

Date de publication : 1 mars 2018

Pagination : 5-11

ISSN : 0994-1908

Référence électronique

Amandine Duluard, «Les Parlements de sciences, outils au service des débats science-société entre jeunes », La Lettre de I'OCIM [En ligne], 176 | 2018, mis en ligne le 01 mars 2019, consulté le 10 octobre 2020. URL : http://journals.openedition.org/ocim/1999 ; DOI : https://doi.org/10.4000/ocim.1999

Ce document a été généré automatiquement le 10 octobre 2020

Tous droits réservés 


\title{
Les Parlements de sciences, outils au service des débats science-société entre jeunes
}

\author{
Amandine Duluard
}

L'auteure tient à remercier Astrid Chevolet, responsable des actions culturelles et Christelle Spettel, responsable de l'action éducative, du Jardin des sciences de l'université de Strasbourg.

Vote d'une proposition en plénière d'un Parlement de sciences à l'université de Strasbourg : le carton de couleur indique l'accord ou non des participants.

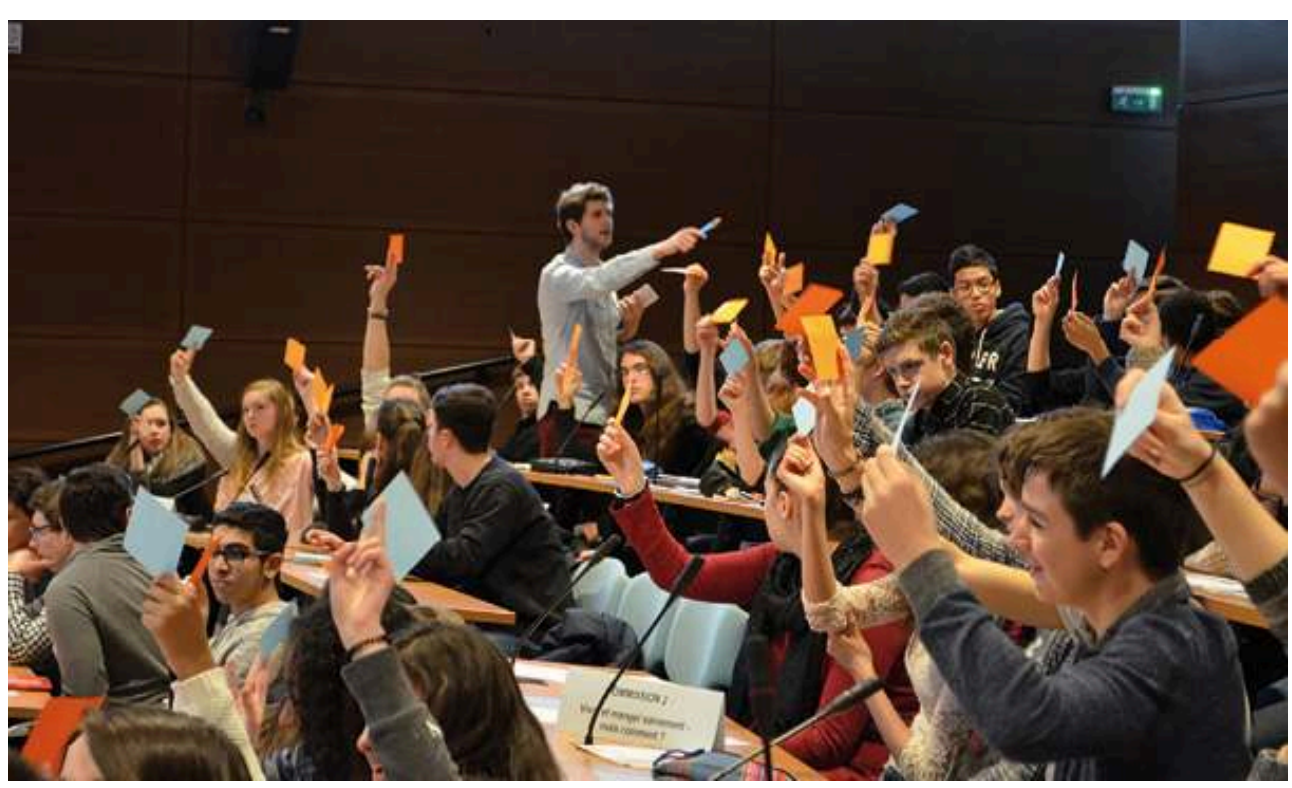

(C) Jardin des sciences-Université de Strasbourg

1 Le Jardin des sciences est le service de médiation culturelle des sciences de l'université de Strasbourg, créé en 1998. Son engagement auprès du public scolaire - notamment 
lycéen - vise à rapprocher le monde de la recherche et ces élèves à travers la mise en débat de questions sciences-société. Dès 2002, voient le jour les cafés scientifiques juniors, qui se déroulent dans les établissements scolaires avec des élèves de collège ou de lycée, puis, en 2009, a lieu le premier Parlement de sciences à l'université de Strasbourg.

2 Cet article revient sur huit années d'expérience dans l'organisation de débats avec et pour les jeunes dans le cadre des Parlements de sciences. Notre objectif est d'y présenter le projet et la manière dont nous l'avons conçu, en soulignant ce que nous pensons être ses principaux bénéfices pour les publics et en revenant sur les difficultés rencontrées lors de sa mise en œuvre. Pour ce faire, nous nous baserons sur les observations empiriques réalisées en tant qu'organisateurs et sur les retours recueillis au fil des années auprès des élèves, enseignants et intervenants.

\section{Le concept}

3 Les Parlements de sciences imitent la structure des processus parlementaires et l'appliquent à des thématiques de l'actualité scientifique. À l'instar des députés, les élèves travail-

4 lent sur des sujets en commissions et rédigent des résolutions qu'ils présentent et défendent lors d'un débat en assemblée appelé aussi séance plénière. En général, les discussions sont clôturées par un vote par lequel les élèves sont amenés à approuver ou rejeter les propositions émises. Le travail en commission couvre approximativement deux tiers du temps de l'événement et la séance plénière le dernier tiers.

5 Ces Parlements rassemblent à chaque édition entre 60 et 90 élèves, répartis en quatre ou cinq commissions de 15 à 20 élèves; les participants sont le plus souvent issus de plusieurs établissements alsaciens. D'une durée d'une ou deux journées, ces événements ont lieu au sein de l'université de Strasbourg.

6 Les thématiques pouvant y être abordées sont variées : bioéthique, agriculture, ville, eau, climat... Le thème choisi est décliné en quatre à cinq sous-thèmes, qui seront étudiés par autant de commissions. Chaque commission bénéficie de la présence d'un spécialiste issu généralement d'un laboratoire universitaire ou du CNRS, ainsi que d'un modérateur, qui anime les débats.

\section{Les objectifs}

$7 \quad$ Les Parlements de sciences se donnent plusieurs objectifs.

8 Tout d'abord, ces événements souhaitent encourager les jeunes à construire leurs propres opinions en prenant en compte les divers avis sur des questions controversées. Ils visent à les rendre acteurs et force de proposition dans les débats de société. Ils constituent par ailleurs une reproduction simplifiée des processus de prise de décision parlementaire et peuvent à ce titre aider les jeunes à mieux cerner les mécanismes et instances de notre démocratie. Ces Parlements de sciences se révèlent également de belles occasions de mobiliser les apprentissages scolaires dans un con-

texte extérieur et de les enrichir par le biais des rencontres d'experts ou d'autres élèves. Enfin, ils font appel à des compétences transversales essentielles à de futurs 
adultes et citoyens, comme l'expression orale, la capacité à argumenter ou encore le respect et l'écoute de l'autre.

D'autre part, l'université se veut à ces occasions un lieu ouvert et d'échanges où il n'est pas seulement question de produire des savoirs et de les transmettre de manière unilatérale. La présence d'intervenants spécialistes de divers domaines met aussi en évidence la variété et la richesse des recherches menées en son sein. Ces événements peuvent ainsi permettre aux élèves de mieux appréhender l'environnement universitaire à une période cruciale de leur orientation.

Les différents Parlements de sciences organisés et le contexte de programmation

\begin{tabular}{|r|l|l|}
\hline \multicolumn{1}{|l|}{} & Thàme & Contexte \\
\hline $20 / 11 / 2009$ & La bioéthique & Dans le cadre du projet européen 2WAYS \\
\hline $23 / 02 / 2012$ & Les biotechnologies & Dans le cadre du projet européen Xplore Health \\
\hline $29 / 05 / 2013$ & L'eau & Année internationale de la coopération dans le domaine de l'eau \\
\hline $14 / 04 / 2014$ & La ville de demain & Projet Debate Science ! \\
\hline $27 / 05 / 2014$ & L'eau en questions & \\
\hline $15 / 12 / 2014$ & L'agriculture & \\
\hline $30 / 01 / 2015$ & La bioéthique & Partenariat avec le Forum Européen de Bioéthique de Strasbourg \\
\hline $27 / 03 / 2015$ & La ville de demain & \\
\hline $28 / 05 / 2015$ & L'eau & \\
\hline $20 / 11 / 2015$ (reporté & Simulation de négociations \\
au $18 / 01 / 2016)$ & climatiques & Label "COP 21" attribué par le comité de pilotage ministériel de la COP 21 \\
\hline 01 et 02/02/2016 & Le futur de l'être humain & Projet Debate Science ! \\
\hline $26 / 04 / 2016$ & La ville de demain & \\
\hline $12 / 12 / 2016$ & $\begin{array}{l}\text { Les ateliers de la créativité - } \\
\text { développement durable }\end{array}$ & Partenariat avec le Vaisseau, dans le cadre de l'exposition le Lab'Oh \\
\hline & & \\
\hline
\end{tabular}

\section{L'historique}

\section{Les débuts ${ }^{1}$}

Le Parlement de sciences est un concept introduit en 2001 par une association berlinoise, Wissenchaft im Dialog, avec pour objectifs de promouvoir l'échange et le dialogue entre scientifiques et élèves, sensibiliser les jeunes à des sujets scientifiques complexes et susciter leur appétence pour les sciences et la recherche.

Ce concept a ensuite été repris à travers l'Europe et initié à Strasbourg en 2009, dans le cadre du projet européen 2 WAYS ${ }^{2}$. Un des volets de ce projet consistait à organiser des Parlements de sciences avec des partenaires locaux dans diverses villes européennes, puis à mettre en œuvre un événement final avec des jeunes représentants de chaque ville, fin 2010 à Bruxelles. 24 partenaires, dont le Jardin des sciences, issus de 17 pays européens, ont ainsi pris part au projet. Tous ont bénéficié de l'expérience de Wissenschaft im Dialog et ont reçu un cahier des charges très complet pour les guider dans l'organisation du Parlement local.

En 2012, le Jardin des sciences participe au projet européen Xplore Health ${ }^{3}$, qui vise à développer de nouveaux outils d'éducation informelle dans le domaine de la recherche biomédicale. Un nouveau projet de Parlement de sciences est alors mis en place à Strasbourg, s'appuyant en partie sur des ressources développées dans le cadre du projet : vidéos de sensibilisation, outils d'aide à la décision comme les jeux PlayDecide ${ }^{4} .$. 

une nouvelle opportunité pour le Jardin des sciences d'organiser un Parlement de sciences localement. Hors du champ du travail en réseau européen, de son appui, mais aussi de ses contraintes, le Jardin des sciences initie sa réflexion sur un format propre susceptible de répondre aux spécificités des contextes académique et éducatif locaux. La même année, afin de développer cette action, une demande de financement $\operatorname{IdEx}^{5}$ est construite avec les Parlement de sciences comme projet phare : le Programme ATOUTS.

Défense au pupitre d'une résolution lors de la séance plénière

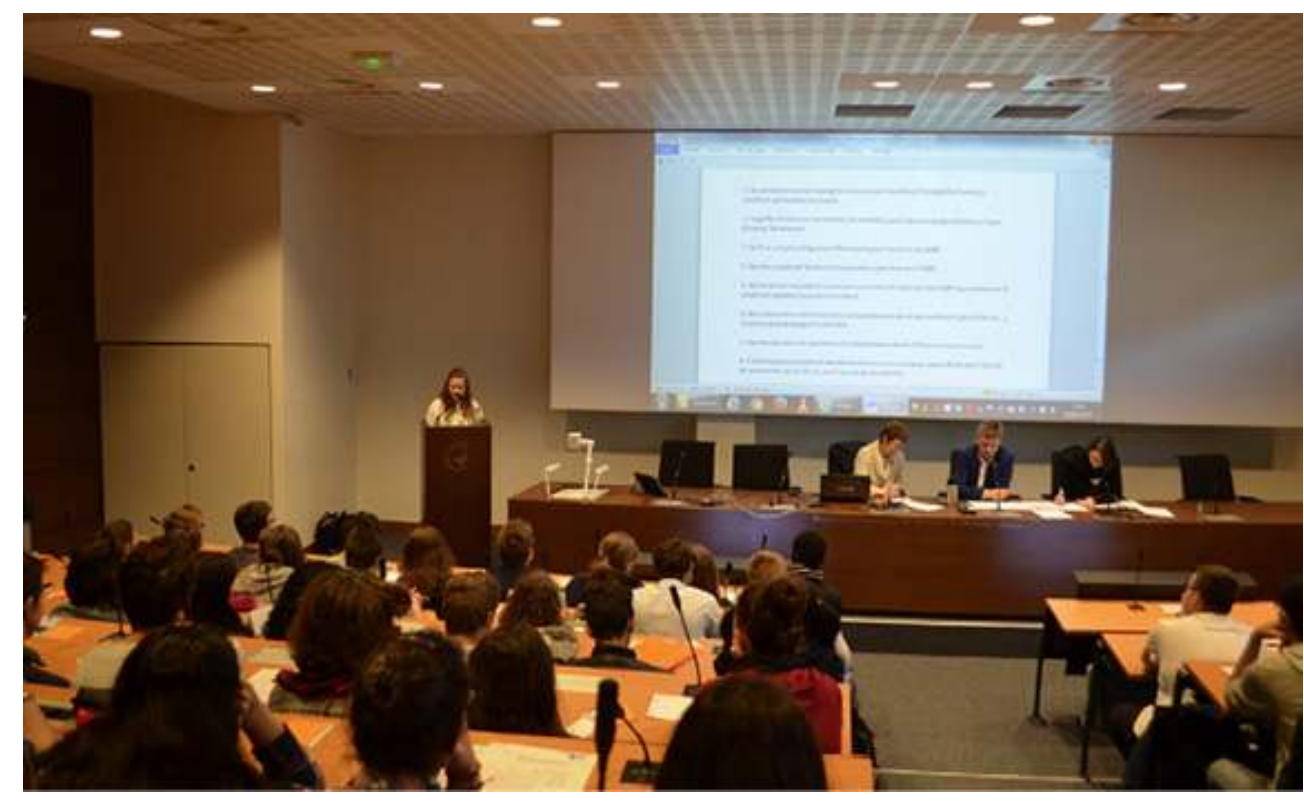

(c) Jardin des sciences-Université de Strasbourg

\section{Les années ATOUTS}

La mise en place du Programme ATOUTS fin 2013, bénéficiant du soutien financier des Investissements d'Avenir, a permis le recrutement de deux personnes à temps complet pour une durée de deux ans et demi et une montée en puissance des Parlements de sciences ${ }^{6}$.

16 La fréquence des événements a considérablement augmenté, avec quatre journées par an programmées en 2015 et 2016. En tout, près de 700 lycéens de la Seconde à la Terminale, issus essentiellement des filières générales et technologiques et provenant de différentes communes alsaciennes, ont pu bénéficier de l'expérience. Cette montée en puissance s'est également traduite en termes de contenus. De nouvelles thématiques ont pu être proposées et des questions relevant des sciences humaines et sociales ont été intégrées aux thèmes débattus. Cela a conduit à une ouverture des événements aux enseignants d'Histoire-Géographie, de Français, de Sciences économiques et sociales...

17 L'instauration d'une régularité dans la mise en place de ces événements a aussi donné l'opportunité de repenser leur déroulement et d'identifier des axes d'amélioration à travers l'observation et les échanges avec les différents participants. Il s'agissait en particulier de dynamiser les débats et de rendre les élèves plus impliqués. Des 
évolutions dans l'organisation et le déroulement des Parlements de sciences ont été testées, puis écartées ou retenues suivant le résultat obtenu : durée du Parlement, planning de l'événement, préparation des modérateurs et des experts, choix du président de séance plénière... Cela a également conduit au développement d'événements au format "dérivé" : la simulation de négociations climatiques et les Ateliers de la créativité. Tout en conservant une structure en deux phases, travaux de groupes puis séance plénière, une coloration différente leur a été donnée. Dans le premier cas, le jeu de rôles reproduisant la structure d'une négociation internationale sur le climat permettait de se focaliser sur le débat et l'argumentation de positions données ; dans le second, l'accent était plutôt mis sur le processus collectif de création d'idées.

18 En parallèle, le réseau d'acteurs européens investis dans l'organisation de Parlements de sciences - dont le Jardin des sciences fait partie - s'est renforcé, grâce au projet Debate science! European Student Parliaments (EUSP) ${ }^{7}$, dont deux éditions ont eu lieu, en 2013-2014 et 2015-2016. À l'instar de 2WAYS, ce projet contenait une phase de Parlements de sciences locaux, organisés par chacun des partenaires, et un événement final européen, réunissant des jeunes de chaque ville.

\section{Le Programme ATOUTS}

Porté par le Jardin des sciences de l'université de Strasbourg dans le cadre des Investissements d'Avenir, entre 2013 et 2016, ATOUTS (A Taste Of University for TeenS) visait à stimuler l'intérêt des scolaires, du cours élémentaire au lycée, pour la recherche et les sciences et les familiariser avec le monde universitaire.

Ce programme visait à développer une politique innovante en matière de diffusion de la culture scientifique et technique auprès des scolaires à l'université, par le biais d'activités incitant les jeunes à découvrir la démarche scientifique et leur permettant d'investir l'université pour mieux en saisir la complexité et la richesse. Il entendait aussi contribuer à la formation des futurs citoyens en leur donnant des clés pour prendre part au dialogue science-société.

Il s'appuyait sur trois manifestations : les Parlements de sciences et Science Lab pour les lycéens, ainsi que Kids University pour les écoliers et collégiens.

\section{Le choix des thèmes et sous-thèmes}

Sept thèmes différents ont été abordés dans les Parlements de sciences. Quelques-uns ont été proposés à plusieurs reprises, en modifiant plus ou moins le contenu des sousthèmes; certains ont été choisis par notre équipe, d'autres correspondaient à un thème commun au projet européen dans lequel l'événement s'inscrivait. 
La prise de parole des élèves durant la séance plénière est facilitée par la présence de micros dans la salle.

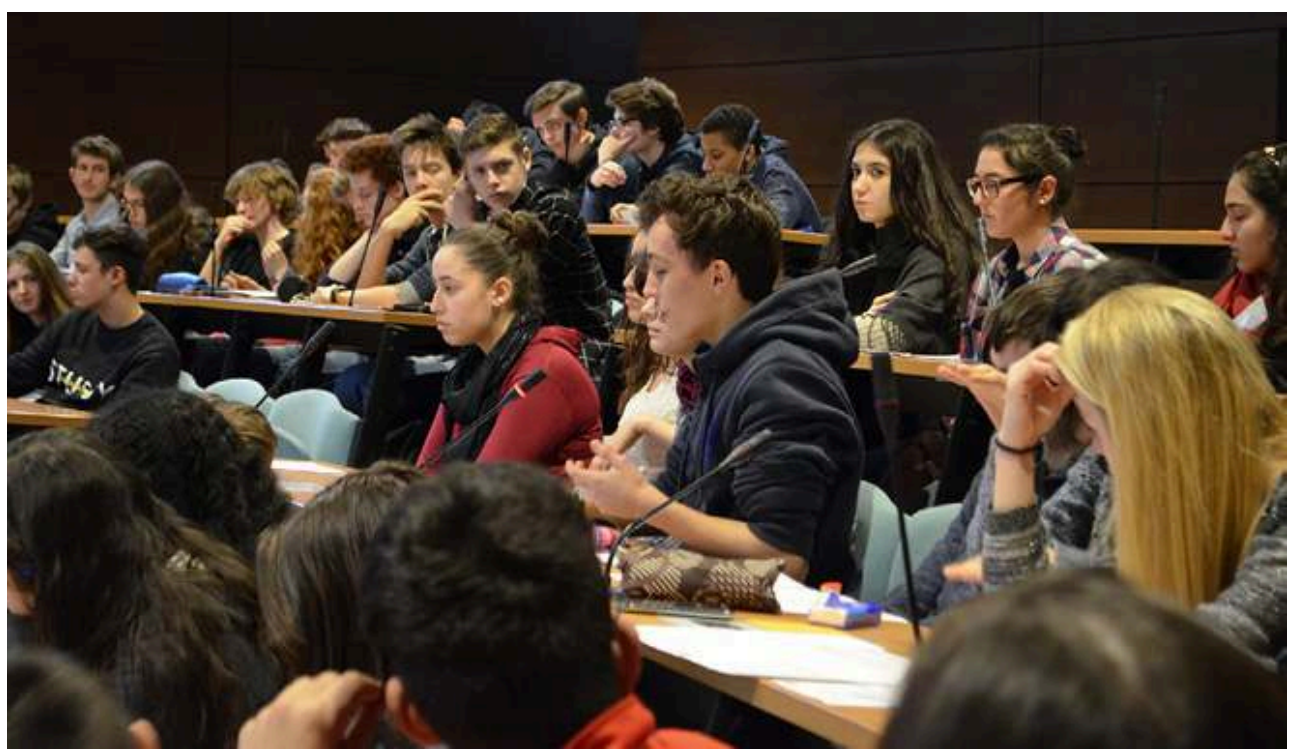

(c) Jardin des sciences-Université de Strasbourg

20 L'analyse de cet échantillon nous a permis d'extraire quelques critères de choix des thèmes et des sous-thèmes de discussion. Les caractéristiques d'un thème pertinent sont selon nous : un lien avec l'actualité ; un caractère prospectif ; une implication dans plusieurs disciplines ; un recouvrement avec des points de programme scolaire de lycée.

21 Les deux premiers critères offrent un véritable potentiel pour capter l'intérêt des jeunes; les deux suivants encouragent les enseignants à s'impliquer dans le projet ou permettent de justifier le temps passé en classe en préparation. Enfin, un thème doit être suffisamment large pour être décliné en plusieurs sous-thèmes, eux-mêmes pas trop pointus. Un thème fonctionnant bien est par exemple "la Ville de demain".

22 Une fois le thème défini, les sous-thèmes déclinés doivent :

23 - être accessibles aux élèves ciblés d'un point de vue conceptuel, pour prévenir une approche purement émotionnelle ;

24 - être reliés au quotidien des élèves ou correspondre à un sujet dans lequel ils peuvent se sentir acteurs ;

25 - accepter des lignes de tension sortant du schéma pour/contre (par exemple un angle bénéfices/risques/limites) ;

26 - éviter de heurter la sensibilité des jeunes ou de toucher à des expériences personnelles douloureuses.

27 On peut par exemple citer les sous-thèmes abordant l'agriculture urbaine ou la participation citoyenne, développés dans le cadre du thème "la Ville de demain". Enfin, de manière plus pratique, il doit être possible d'en trouver un spécialiste capable de s'adresser aux jeunes, travaillant dans un périmètre géographique limité. 


\section{La place des adultes}

Nous avons observé que le rôle donné aux adultes est crucial dans le processus ; les enseignants, modérateurs, spécialistes, doivent accompagner les débats des élèves. Leur réflexion ou leur parole ne doit pas se substituer à celles des jeunes, que cela soit par habitude ou pour combler d'éventuels silences. De même, la présence d'adultes spectateurs dans un travail en petit groupe est à éviter, les élèves risquant de se sentir jugés.

\section{Le modérateur : un rôle essentiel, mais délicat}

Chaque groupe d'élèves ou commission est encadré par un modérateur. Son rôle est essentiel dans la mise en place des échanges. Il doit instaurer un climat de confiance où la parole est libre, écoutée et respectée ; ceci est d'autant plus important que les élèves seront souvent amenés à donner leur avis personnel.

Le rôle de modérateur a parfois été confié à des enseignants. Ceux-ci connaissent bien les élèves et sont habitués à les dynamiser. La plupart ne sont cependant pas formés à l'animation de débats et auraient besoin d'être accompagnés pour mener la tâche à bien. Le recrutement d'un intervenant professionnel extérieur peut constituer une bonne alternative si l'on dispose de moyens financiers, mais soulève un autre questionnement : quel rôle donner aux enseignants dans ce cas ? Nos expériences dans ces conditions n'ont pas été très concluantes : les enseignants ne trouvaient pas leur place dans les commissions ou se sentaient désinvestis du projet. Une piste pourrait être dans ce cas de proposer un programme spécifique aux enseignants, avec des temps d'échanges entre enseignants et éventuellement avec les organisateurs, autour par exemple de la valorisation du projet dans leurs établissements ou plus largement, des propositions d'amélioration.

Sortir du cadre pour initier les réflexions, par exemple à l'aide du dessin.

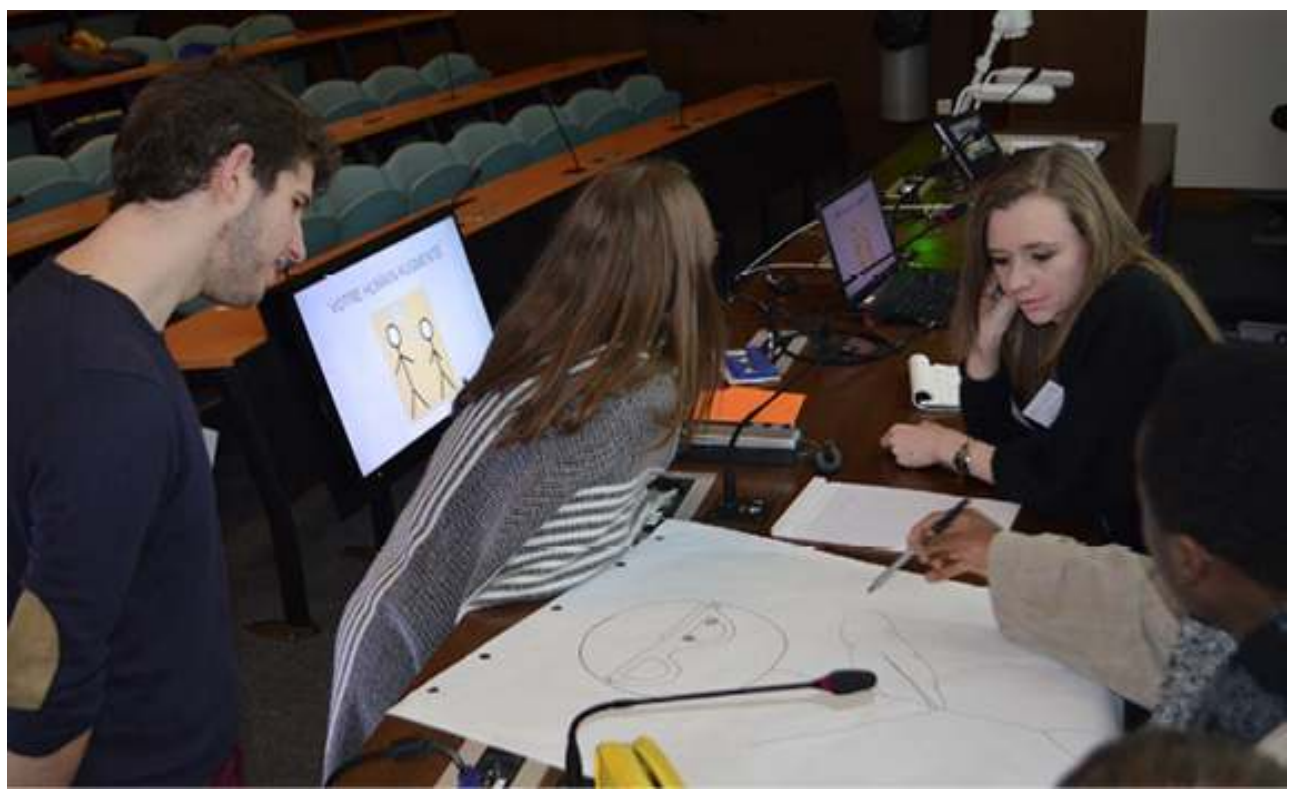

(c) Jardin des sciences - Université de Strasbourg 
Enter arriver à instaurer un débat en séance plénière avec 70 à 100 personnes dans un cadre codifié est un vrai défi, dont nous avons fait l'expérience avec plus ou moins de succès. Il faut à la fois rendre le débat accessible à tous et encourager les échanges, tout en étant capable de recentrer les discussions. Nous faisons à présent appel à un comédien d'improvisation, féru de sciences et attaché à la démarche et au questionnement scientifiques, qui parvient à trouver un subtil équilibre entre jeu de rôle et rigueur. Il incarne la posture très sérieuse du président de séance tout en parvenant à mettre les élèves à l'aise pour s'exprimer ; restant dans ce rôle, il peut aussi parfaitement faire face à toute situation sans se désarçonner et remobiliser les élèves. Enfin, il s'avère exigeant sur le fond, la précision et la source des arguments avancés et encourage ainsi la qualité des échanges.

\section{Le spécialiste : une intervention minimale dans les débats}

Une difficulté que nous avons rencontrée à plusieurs reprises est la tendance des élèves à répéter les dires d'un spécialiste, sans se les approprier voire parfois sans en comprendre le sens. La présence des experts n'est, selon notre expérience, pas indispensable tout au long du processus. Si une intervention de leur part est nécessaire pour apporter des éléments scientifiques aux discussions des jeunes, ces derniers doivent pouvoir bénéficier de moments durant lesquels ils se sentent libres de mener leur propre réflexion et de prendre du recul par rapport à la position de l'expert, pas nécessairement représentative du débat d'idées. Dans cette optique, nous essayons de définir des temps précis dans le programme de la journée, destinés à l'échange avec un spécialiste, d'une durée de $1 \mathrm{~h} 30$ à $2 \mathrm{~h}$.

\section{Ils parlent de leur expérience}

\section{$\underline{\text { Les élèves }}$}

"La manifestation était superbe. Elle permet de nous ouvrir un peu plus l'esprit et de réaliser ce qui se passe réellement dans le monde. Les jeunes ne font pas vraiment attention à la ville d'aujourd'hui et ce à quoi elle pourrait ressembler demain, mais cette manifestation nous a permis de nous impliquer dans cela et de changer certaines mentalités et c'est une très bonne chose".

\section{$\underline{\text { Les enseignants }}$}

- "Les élèves peuvent affirmer leurs positions mais aussi entendre et écouter ce que les autres disent, les arguments qu'ils déploient. Tous ne participent pas aux tables rondes, mais tous ont un avis. [...] Certains s'engageront peut-être après dans la vie associative ou affirmeront plus facilement leurs positions".

- "Les élèves ont été mis en situation de réflexion et de travail, et traités avec respect comme des acteurs de la société présente et à venir".

- "À leur âge, les élèves n'ont pas eu beaucoup d'occasions d'exprimer leurs idées en dehors d'une salle de classe [...] C'est l'occasion pour eux de se confronter à d'autres points de vue, [...] ainsi qu'une bonne occasion de s'exprimer en public". 


\section{Les chercheurs}

- "Cette manifestation est plus qu'un exercice, elle sensibilise sur des questions d'actualité et contribue à une ouverture (d'esprit) à travers les nombreux aspects qui sont proposés pour discussion dans les différentes commissions".

- "Il y un projet clair, des supports, un cadre de réflexion et une animation conséquente. La manifestation est très mobilisatrice et les participants sont vraiment investis!".

- "C'est une très bonne opportunité pour les élèves d'apprendre ce que c'est la réalité d'un débat où tout le monde n'a pas forcément le même avis mais où il faut trouver des consensus".

\section{Prendre les élèves au sérieux et donner du sens à l'exercice}

Nous pensons que le cadre de la manifestation a également son importance : des salles en nombre suffisant, avec un équipement adapté et confortable, un accueil officiel par une personnalité politique ou universitaire, l'organisation de pauses café... Tout ce qui peut rappeler le contexte de travail des adultes contribue à renforcer l'impression chez les élèves qu'ils seront pris au sérieux. Nous essayons également de veiller à la cohérence entre le cadre que l'on souhaite instaurer et le discours tenu, par exemple en parlant de "collation", plutôt que de "goûter".

Si l'exercice proposé par le Parlement de sciences contribue à leur formation de futurs adultes et citoyens, les élèves évoquent souvent le besoin de donner un sens plus immédiat au travail qu'ils fournissent. Plus rarement, certains qui avaient pourtant participé activement aux échanges, ont déploré le fait que leurs propositions ne serviraient à rien. Ces revendications sont à notre sens légitimes et nous tentons d'y répondre de deux manières complémentaires. D'une part, en abordant ouvertement cette question lors de l'événement, et d'autre part en produisant durant la journée un document de synthèse faisant sens pour les élèves comme pour les partenaires à qui il pourra être transmis. Après essai de différents formats, nous avons finalement opté pour un document de type "proposition de résolution". Celui-ci mentionne des clauses introductives et opérationnelles votées après débat et constitue un livrable disponible immédiatement, émanant des élèves et reflétant la position du groupe. 
Les activités brise-glace, temps indispensable pour instaurer la confiance entre les participants au sein des commissions.

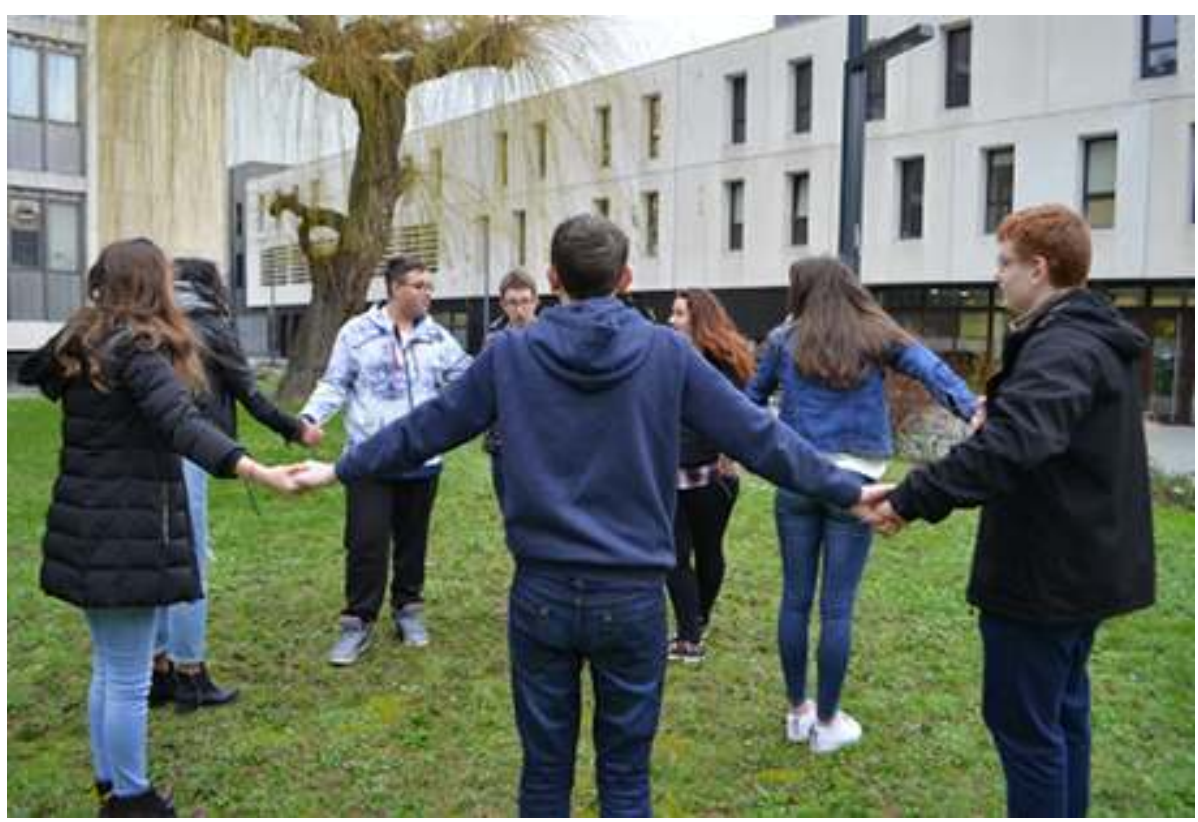

(c) Jardin des sciences-Université de Strasbourg

\section{Le rôle des partenaires}

Le soutien des institutions nous semble un aspect primordial dans un tel événement. Nous mettons à profit notre collaboration avec le Rectorat de l'Académie de Strasbourg, qui appuie nos appels à participation au sein des établissements et rend possible une implication importante des enseignants dans les projets.

Au fil des années, nous avons également ressenti la nécessité et la pertinence de nouer des liens avec les institutions locales (Ville de Strasbourg, Région Alsace, puis Région Grand-Est) pour des raisons financières mais aussi pour leur potentiel de valorisation $\mathrm{du}$ travail des jeunes. Les élus de ces collectivités concernés par les thématiques abordées sont ainsi invités de manière presque systématique afin d'être les récipiendaires de la résolution produite par les élèves. Toutefois, si les élus se prêtent volontiers au jeu, l'action n'en demeure pas moins symbolique.

\section{Préparer les parties prenantes}

Le bon déroulement d'un Parlement implique une préparation attentive des participants : élèves bien sûr, mais aussi enseignants, modérateurs et spécialistes.

Notre préparation repose actuellement sur de la documentation : dossiers documentaires ou sitographies, parfois assortis de supports pédagogiques, pour la préparation thématique des élèves et des modérateurs, fiches méthodes et cahier des charges détaillé pour le modérateur. Ces éléments sont présentés et discutés avec les enseignants et les modérateurs lors de rencontres. Mais cet accompagnement minimal dans la prise en main des outils s'avère parfois insuffisant et induit une préparation et une aisance inégales à la fois des élèves et des enseignants/modérateurs. 

ont été organisés, réunissant plus de 900 élèves. Le dispositif semble avoir un réel impact sur ces derniers.

41 Ils s'enrichissent de leur expérience du débat contradictoire : "si l'on est intimement persuadé de quelque chose, qui nous semble évident, il n'en va pas toujours de même pour tout le monde... Et il faut apprendre à écouter posément les arguments contraires, les comprendre, et savoir en tenir compte dans son jugement", indique un élève. La prise au sérieux de leur parole est très valorisante, comme le soulignent ces autres élèves : "Le débat a été notre partie favorite, pouvoir s'exprimer et se faire écouter sérieusement était enrichissant". Les enseignants partagent un avis très positif sur la manifestation et ses bénéfices sur les élèves, comme en témoigne une enseignante : "L'expérience est très enrichissante pour les élèves et peut améliorer leur estime propre, leur permettre de se projeter dans des études universitaires"; un autre indice de l'engouement des enseignants est leur volonté de réinscrire une classe l'année suivante.

$42 \mathrm{Au}$ fil des éditions, des difficultés ont pu être identifiées par les organisateurs ou soulevées par les participants : timidité des échanges entre élèves ou avec les spécialistes, manque de valorisation du travail des jeunes, disparité d'implication et d'aisance des enseignants et des élèves, difficulté des élèves à prendre du recul vis-à-vis de la parole du spécialiste. Notre réflexion pour y apporter des réponses est toujours en cours et se nourrit de nos observations, des retours des participants et d'essais de nouveaux formats.

Enfin, l'organisation de tels événements représente un coût non négligeable (chargé de projet, location de salles, rémunération de modérateurs, repas...) qui impacte directement sur leur fréquence et qui nécessite des partenaires financiers dans la durée. 
Travaux en commission dans une disposition "table ronde"

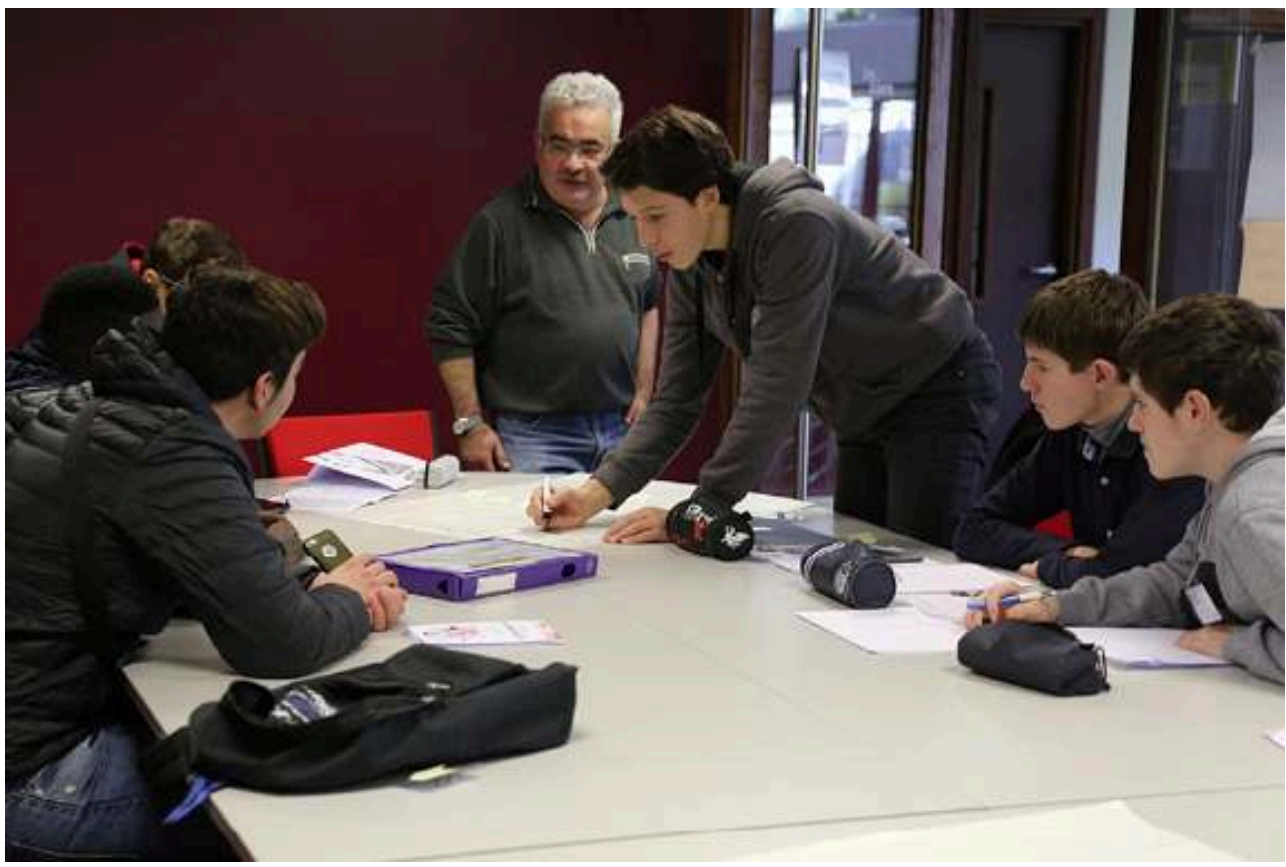

(c) Université de Strasbourg/C. Schröder

\section{Et maintenant}

Après une phase de prise en main du format et une phase de développement important, les Parlements de sciences sont devenus une action emblématique du Jardin des sciences, plébiscitée par les enseignants et encouragée par les institutions locales. Si l'IdEx a servi de levier, il reste maintenant à s'assurer un financement pérenne.

Notre capacité à créer les conditions de discussion dans nos événements s'est accrue, mais des difficultés et questions demeurent. Pour l'organisation du prochain Parlement au printemps 2018, nous bénéficions du soutien d'une doctorante en sciences de l'éducation, en mission complémentaire de diffusion de l'information scientifique et technique auprès du Jardin des sciences ${ }^{8}$. Il s'agira d'affiner la réflexion déjà menée et de porter un regard nouveau sur nos pratiques. Nous envisageons de proposer des améliorations dans la préparation des élèves et des enseignants (nouvelles pratiques, supports pédagogiques...) et espérons être en mesure de réaliser une évaluation des apports pour les élèves, allant au-delà du questionnaire de satisfaction.

Plus largement, notre pratique du débat avec les jeunes nous interroge. Dans cette optique, trois autres doctorants missionnés renforcent l'équipe du Jardin des sciences en 2017-2018 sur des projets en lien avec la thématique "débats et jeunes". Une doctorante va accompagner des équipes pédagogiques dans la mise en place de simulations de négociations climatiques dans leur établissement scolaire et deux doctorants travaillent à l'amélioration de notre dispositif de cafés scientifiques juniors.

47 La réflexion sur les formats et la précision de bonnes pratiques pour créer les conditions de discussion est donc bien active dans nos murs, mais elle gagnerait encore à bénéficier de retours d'expériences d'autres structures ; par ailleurs, rien ne s'oppose à un essaimage des Parlements de sciences en région ou en France auprès de 
partenaires cherchant à développer des actions de mise en débat des questions sciencesociété auprès des jeunes.

\section{NOTES}

1. Ces projets ont été coordonnés par Christelle Spettel, responsable de l'action éducative au Jardin des sciences.

2. 2WAYS est un projet européen porté par l'association Eusea, qui a été actif du 01/01/2009 au 31/12/2010. Plus d'informations sur le projet sur le site du Service communautaire d'Information sur la recherche et le développement de la Commission européenne : cordis.europa.eu.

3. Xplore Health est un projet européen coordonné par Barcelona Science Park entre 2010 et 2012. Son objectif était de développer un portail éducatif européen, pour des jeunes de 8 à 18 ans, portant sur des sujets de recherche de pointe dans le domaine de la recherche biomédicale. Le portail est toujours actif à l'heure actuelle : www.xplorehealth.eu/. Plus d'informations sur le projet sur le site du Service communautaire d'Information sur la recherche et le développement de la Commission européenne : cordis.europa.eu.

4. Les jeux de débat PlayDecide permettent d'engager des discussions sur des sujets controversés. Composés d'un plateau de jeu et de cartes diverses (témoignages, infos, thèmes de discussions), ils offrent aux joueurs la possibilité d'appréhender différentes positions et de clarifier leur opinion sur les sujets abordés : www.playdecide.eu.

5. L'initiative d'excellence (IdEx) est un programme des Investissements d'Avenir dont le but est de créer en France des ensembles pluridisciplinaires d'enseignement supérieur et de recherche de rang mondial. L'université de Strasbourg en est lauréate depuis 2012.

6. Les deux personnes recrutées pour assurer la mise en place du programme étaient Clémence Bohn, assistante chargée de médiation scientifique et Amandine Duluard, chargée de projet en médiation scientifique.

7. Initié par Wissenschaft im Dialog, ce projet a fédéré une quinzaine d'acteurs associatifs et universitaires d'une dizaine de pays européens. Les Parlements finaux se sont déroulés en lien avec la conférence internationale EuroScience Open Forum (ESOF), à Copenhague en juin 2014 et à Manchester en juillet 2016 : www.student-parliaments.eu.

8. Parallèlement à sa mission de recherche, le doctorant peut effectuer d'autres activités, rémunérées ou non. En particulier, un doctorant contractuel peut exercer une "mission complémentaire", activité hors recherche rémunérée comptant pour un sixième de son temps annuel de travail. Plusieurs types de missions existent, parmi lesquelles la mission complémentaire de diffusion de l'information scientifique et technique. 


\section{RÉSUMÉS}

Comment faire en sorte que les jeunes s'impliquent davantage dans les échanges autour de l'actualité scientifique ? Les Parlements de sciences mis en place par l'université de Strasbourg proposent une réponse à cette interrogation : après une présentation de ces dispositifs et une première évaluation leurs apports, l'auteur ouvre des pistes pour compléter la réflexion et développer ce moyen de mise en débat des questions science-société.

\section{INDEX}

Mots-clés : débats, science-société, médiation

\section{AUTEUR}

\section{AMANDINE DULUARD}

Chargée de projet en médiation scientifique au Jardin des sciences de l'université de Strasbourg. amandine.duluard@unistra.fr 